\title{
Prevalencia de sobrepeso y obesidad, hábitos alimentarios y actividad física y su relación sobre el rendimiento académico \\ Prevalence of overweight and obesity, exercise, and dietary habits, and their relation with academic achievement \\ Álvaro Iglesias, Elena Planells, Jorge Molina López \\ Universidad de Huelva (España)
}

\begin{abstract}
Resumen. El presente estudio tuvo como objetivo determinar la prevalencia de sobrepeso y obesidad así como evaluar la práctica de actividad física y pautas alimentarias en niños escolares de $3^{\circ}$ Ciclo de Educación Primaria y su relación con el rendimiento escolar. Un total de 115 niños de 5 y $6^{\circ}$ de Educación Primaria fueron seleccionados en escuelas públicas de Bollullos del Condado (Huelva). La composición corporal se determinó mediante impedancia bioeléctrica (Tanita MC-980), la actividad física mediante el cuestionario PAQA, los hábitos alimentarios mediante un cuestionario de frecuencia de consumo, hábitos y prácticas de alimentación para escolares y el rendimiento académico con las calificaciones medias del trimestre anterior. Se observó una mayor práctica de actividad física y mayor masa muscular y ósea por parte de los chicos que las chicas. Las cifras de sobrepeso y/o obesidad y/o porcentaje graso resultaron ser elevadas y el consumo de alimentos básicos reducido, observándose relación entre un bajo consumo de frutas, verduras y lácteos con un peor rendimiento académico. Existe la necesidad de fomentar una educación en hábitos saludables, con la finalidad de aumentar la práctica de actividad física y mejores hábitos alimentarios, repercutiendo así sobre el rendimiento académico.
\end{abstract}

Palabras clave. Rendimiento Académico; Actividad Física; Adolescentes; Hábitos Alimentarios.

Abstract. The aim of this study was to determine the prevalence of overweight and obesity, as well as to evaluate physical activity and food frequency patterns in school-aged children from the 3rd cycle of Primary Education, and their relationship with academic achievement. A total of 115 students were selected from 5th and 6th grades of public primary schools in Bollullos del Condado (Huelva). Body composition was assessed through Tanita MC-980, physical activity through the PAQ-A questionnaire, dietary habits using a food frequency consumption questionnaire, and academic performance by reviewing students' grades from the previous term. Results showed greater physical activity practice together with greater muscular and bone mass in boys compared to girls. Regarding fat percentage, a high prevalence of overweight and/or obesity was observed. The food consumption analysis revealed a reduced consumption of basic groups of food in contrast with unhealthier food; a relation between lower consumption of fruits, vegetables and dairy products and lower academic performance was observed. There is a need to promote healthy habits education, with the aim of increasing the practice of physical activity, as well as to improve dietary habits, which have an impact on academic performance.

Keywords. Academic achievement, physical activity, adolescents, dietary habits.

\section{Introducción}

La obesidad está considerada uno de los problemas más serios en las últimas dos décadas. La Organización Mundial de la Salud (OMS) ha creado el término «globesity» para hacer referencia al aumento progresivo que ha experimentado la obesidad en los últimos 40 años (Angosto, 2015). En 2017, la OMS analizó la evolución del IMC y obesidad desde 1975 hasta 2016 mostrando que la obesidad infantil y adolescente se multiplicó por 10, pasando de 11 millones a 124 millones (Organización Mundial de la Salud, 2016). Igualmente, 213 millones de jóvenes presentaban sobrepeso sin llegar a alcanzar el umbral de obesidad. La OMS establece el inicio de la adolescencia a los 10 años (Organización Mundial de la Salud, 2018). Según esta clasificación los alumnos de $5^{\circ}$ y $6^{\circ}$ curso de Primaria podrían incluirse dentro de esta complicada etapa, concretamente en la adolescencia temprana. En España, encontramos un 4,7\% de población adolescente que sufre obesidad, existiendo una diferencia considerable entre chicos (6,4\%) y chicas (2,9\%) (Inchley, Currie, Jewell, Breda, y Barnekow, 2017).

En lo que respecta a la actividad física (AF), la OMS recomienda que los niños y adolescentes dediquen como mínimo 60 minutos de AF moderada-vigorosa, obteniéndose

Fecha recepción: 04-07-18. Fecha de aceptación: 29-01-19 Benito Alonso Urra Tobar benitourrat@gmail.com beneficios superiores si el tiempo dedicado es superior. Actualmente, se ha descrito que el $81 \%$ de adolescentes con edades entre 11 y 17 años no cumplieron esta recomendación en 2010, siendo las chicas menos activas (84\%) que los chicos (78\%) (Organización Mundial de la Salud, 2018). Algunos de los principales beneficios de la AF en adolescentes han sido referidos a la mejora de la salud cardiovascular, mejorando factores determinantes como la presión sanguínea (Son, Sung, Bharath, Choi, y Park, 2017), la reducción de hasta un 40\% del riesgo de sufrir cáncer asociado a enfermedades crónicas (Tu et al., 2018). Existen también evidencias científicas que remarcan los beneficios de la práctica de AF por provocar mejoras considerables en los procesos cognitivos, además de los procesos de memorización y atención de los adolescentes (Abalde-Amoedo y Pino-Juste, 2016; Guillamón, Canto y López, 2019).

De la misma manera, la alimentación es considerada otro de los pilares fundamentales en los adolescentes. Una alimentación cuyo aporte nutricional sea insuficiente o inadecuado puede perjudicar gravemente su crecimiento y desarrollo (Moreno, 2015). Estudios como los de Gargallo Fernández et al. (2012), han puesto de manifiesto los numerosos beneficios reportados por una buena alimentación. Alguno de los efectos descritos hacen referencia a la reducción del riesgo de padecer alguna enfermedad cardiovascular, diabetes o cáncer. Igualmente, en Andalucía se comprobó que existe una relación positiva entre unas buenas pautas alimentarias y la práctica de AF con mejoras cognitivas en 
adolescentes (Knox y Muros, 2017). Teniendo en cuenta lo anteriormente mencionado, existen estudios que han determinado la adherencia a la dieta Mediterránea sin proporcionar información cuantitativa sobre el grado de adecuación de las pautas alimentarias, atendiendo a la frecuencia de consumo de un grupo de alimentos (Iaccarino Idelson, Scalfi y Valerio, 2017). En este sentido, el estudio de la adecuación desde la perspectiva del análisis de la frecuencia de consumo de alimentos, podría aportar mayor información adicional sobre las pautas alimentarias que los adolescentes reportan basándonos en el modelo de pirámide alimentaria propuesto por la Sociedad Española de Nutrición Comunitaria (SENC) (Sociedad Española de Nutrición Comunitaria, 2017).

Actualmente, el rendimiento académico es uno de los indicadores de calidad del sistema educativo, así como de las materias, asignaturas o áreas curriculares que lo conforman (OECD, 2012). Debido a ello, numerosos estudios han analizado posibles variables y su asociación con el rendimiento académico desde diferentes enfoques y perspectivas (Álvarez-Bueno, et al. 2017). Además, existen estudios (Pérez-Farinós et al., 2017) que han analizado la relación entre el consumo de frutas y verduras y el nivel educativo de estos, observando un menor consumo de frutas y verduras cuando los padres tienen un nivel educativo menor. En otro reciente estudio (Burrows, Goldman, Olson, Byrne y Coventry, 2017), se encontró relación entre un mayor consumo de verduras en la cena con puntuaciones más altas en ortografía y escritura (Burrows, Goldman, Pursey, y Lim, 2017). Sin embargo, a pesar de la evidencia actual, existen resultados contradictorios sobre la relación de las variables comentadas anteriormente. Por tanto, resultan necesarios nuevos estudios que permitan clarificar la relación entre actividad física, hábitos alimentarios y rendimiento académico.

Nuestro estudio hipotetiza que aquellos niños con una mayor práctica de AF y unos buenos hábitos alimentarios presentarán un mejor IMC, lo que repercutirá positivamente en su rendimiento académico. Por tanto, el presente estudio tuvo como objetivo determinar la prevalencia de sobrepeso y obesidad, así como evaluar la práctica de AF y pautas alimentarias en niños escolares de $3^{\circ}$ ciclo de Educación Primaria, y su relación con el rendimiento escolar en Bollullos par del Condado, en la provincia de Huelva.

\section{Metodología}

\section{Participantes}

El presente estudio es un diseño no experimental, observacional, transversal y analítico realizado en Bollullos par del Condado (Huelva) durante los meses de marzo-abril de 2018.

La muestra del estudio estuvo compuesta por un total de 115 jóvenes de $5^{\circ}$ y $6^{\circ}$ de Educación Primaria, con edades comprendidas entre 10 y 12 años. Se seleccionaron alumnos de los colegios Reyes Católicos y Las Viñas, pertenecientes a la localidad de Bollullos par del Condado (Huelva).

El muestreo realizado fue intencional o por conveniencia, ya que se quería obtener una muestra de jóvenes con edades comprendidas entre 10-12 años, para lo que se contactó con distintos centros de la localidad. Las proporciones de varones y mujeres no fueron estadísticamente dis- tintas, ni tampoco existieron diferencias de edad entre ambos sexos. Se invitó a participar a todos los alumnos de ambos cursos, contactando previamente con sus tutores legales a través de un documento informativo, cuyo consentimiento debían traer completo y firmado antes del día elegido para poder participar en el estudio. Los participantes recibieron instrucciones sobre cómo completar los cuestionarios y cómo realizar las pruebas. Toda la información se recopiló durante el horario escolar. No se proporcionaron incentivos a los adolescentes y padres. Un asistente de investigación también estuvo presente para proporcionar orientación sobre la realización de cuestionarios y la determinación de la composición corporal.

\section{Instrumentos}

Las mediciones de composición corporal se tomaron por impedancia bioeléctrica multifrecuencia (Tanita MC-980 Body Composition Analyzer MA Multifrequency Segmental, Barcelona, España). El analizador cumple con las normas europeas aplicables (93/42EEC, 90/384EEC) para su uso en la industria médica. Los participantes fueron informados con antelación de las condiciones requeridas antes de la medición: sin alcohol menos de 24 horas antes de la medición, sin ejercicio vigoroso menos de 12 horas antes de la medición, sin comida o bebida menos de 3 horas antes de la medición, y no hay necesidad de orinar inmediatamente antes de la medición. Todas las mediciones se tomaron simultáneamente durante la mañana en ayunas. Se calcularon las mediciones del peso y del índice de masa corporal, y el análisis compartimental midió la masa grasa, la masa libre de grasa y la masa muscular. Todas las mediciones se tomaron durante dos mañanas, una por cada centro educativo. Para calcular el grado de normalidad respecto al IMC y el porcentaje graso, se utilizó como referencia las curvas de IMC y grasa presentadas por Centers for Disease Control and Prevention (Laurson, Eisenmann, y Welk, 2011; Nagy et al., 2014).

Para la actividad física el instrumento utilizado fue el Cuestionario de Actividad Física para Adolescentes (PAQA/Physical Activity Questionnaire for Adolescents.) previamente validado (Martínez-Gómez, 2009). Los estudios de validación han encontrado que el PAQ-A es altamente confiable. El cuestionario consta de 9 preguntas que miden los niveles de actividad física de los últimos 7 días, las 8 primeras preguntas se utilizan para evaluar el nivel de actividad física, mientras que la última pregunta es para conocer si el alumno estuvo enfermo o algo le impidió realizar actividad física con normalidad. Las puntuaciones para cada pregunta oscilan del 1 al 5, siendo el 1 una actividad física baja y el 5 muy elevada. La puntuación final se obtiene calculando la media de las 8 preguntas evaluadas previamente. Las puntuaciones de corte establecidas para categorizar la AF fue de 2,75 puntos para discriminar entre jóvenes «activos» $\mathrm{y}$ «no activos» (AF moderada-vigorosa > $60 \mathrm{~min}$ / día, AF vigorosa> $30 \mathrm{~min}$ / día y AF ligera > $116 \mathrm{~min} /$ día; respectivamente) (Benítez-Porres, Alvero-Cruz, Sardinha, López-Fernández, y Carnero, 2016).

Los hábitos alimentarios fueron evaluados a través de un cuestionario de frecuencia de consumo de alimentos (Lera, Fretes, González, Salinas y Vio, 2015). El cuestionario consta de 28 preguntas relacionadas con habilidades culinarias (6 
preguntas), la frecuencia de consumo de alimentos (FFQ) (12 preguntas), los hábitos alimentarios (7 preguntas) y gastos en la escuela (3 preguntas). La FFQ se usó para establecer la información sobre el consumo de frecuencia en cada grupo de alimentos. El FFQ se comparó con las recomendaciones propuestas por la SENC (Sociedad Española de Nutrición Comunitaria, 2017), y se expresó como el porcentaje de participantes por debajo, dentro y por encima del número recomendado de raciones para cada grupo de alimentos. En caso de no existir una cantidad recomendada de porciones para un alimento dado porque se consume ocasionalmente, los resultados fueron expresados en raciones consumidas por semana.

Para conocer el rendimiento académico de los escolares se ha utilizado la calificación media de cada alumno en el trimestre previo al estudio, siendo este el segundo de un total de tres trimestres anuales. Las calificaciones fueron entregadas por el propio centro educativo de acuerdo a la Ley de Protección de Datos vigente. La calificación fue categorizada en Suspenso, Aprobado, Notable y Sobresaliente con la finalidad de explorar el grado de asociación entre el rendimiento académico con el resto de variables.

\section{Procedimiento}

Una vez decididos los instrumentos a utilizar, se contactó con los responsables de ambos colegios, y se les entregó la información del estudio que pretendíamos realizar. El siguiente paso fue explicar y entregar los cuestionarios a los alumnos, y a medida que lo iban rellenando iban pasando a una sala aislada para tomarles mediciones. Posteriormente, analizamos todos los datos obtenidos y encontramos nuestros resultados principales.

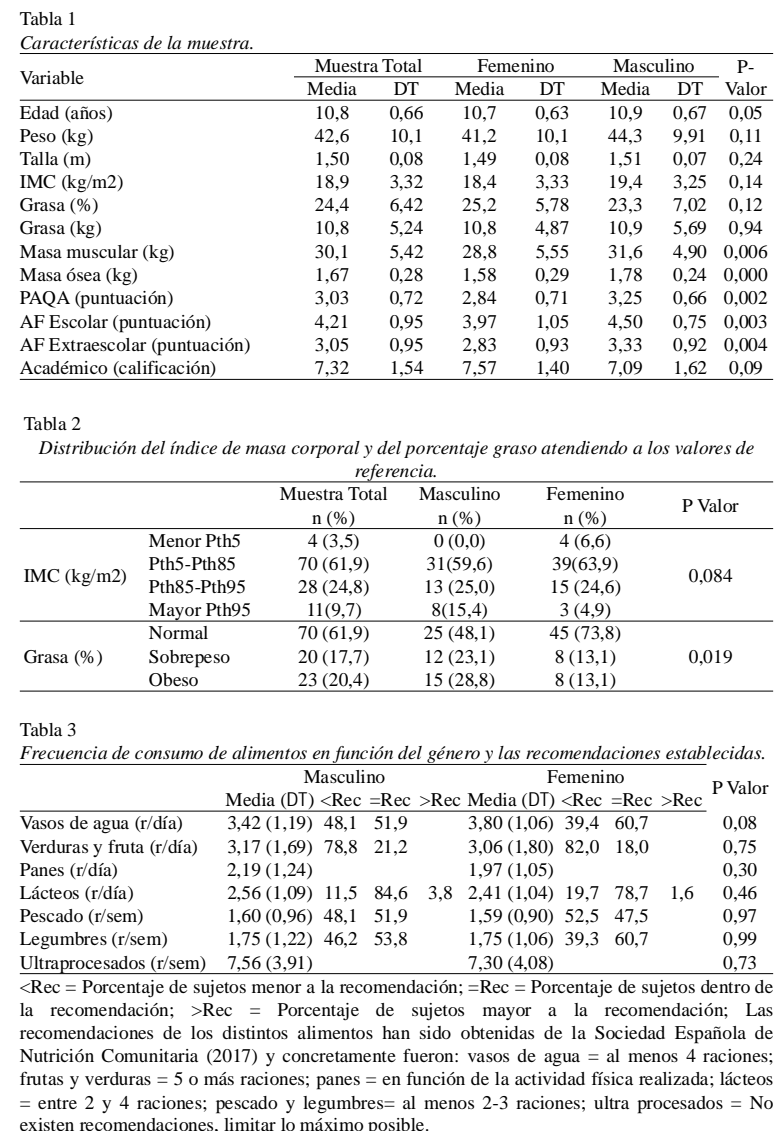

\section{Análisis estadístico de los datos}

Los datos se analizaron usando SPSS 20.0 para Windows (SPSS Inc. Chicago, IL, EE. UU.). Los valores de las variables cuantitativas se expresaron como los valores medios y la desviación estándar para el análisis descriptivo. Para las variables cualitativas se expresó la muestra y el porcentaje de sujetos. La estandarización de las variables se llevó a cabo utilizando la prueba de Shapiro-Wilk con corrección de Lilliefors. La homocedasticidad se determinó mediante la prueba de Levene. Después de verificar que las variables eran normales, los datos se analizaron mediante la prueba $\mathrm{T}$ para muestras independientes con la finalidad de determinar la composición corporal, la frecuencia de práctica de actividad física y la frecuencia de consumo de alimentos en función del sexo. La prueba de Chi-cuadrado mediante el ajuste de Bonferroni se realizó para determinar la distribución de la muestra respecto al índice de masa corporal y el porcentaje graso en función del sexo. Igualmente, se determinó el grado de adecuación de los jóvenes respecto a la FFQ. Finalmente, el modelo lineal multivariado se realizó para determinar el IMC y el sexo sobre los valores medios de práctica de actividad física, la FFQ y el rendimiento académico. Igualmente, el modelo lineal multivariado se realizó para determinar la influencia del rendimiento académico y el sexo sobre la composición corporal, práctica de actividad física y la FFQ. El nivel de significación estadística se estableció en 0.05.

\section{Resultados}

La Tabla 1 expone las características de la muestra de estudio. Los resultados mostraron que los niños presentaban niveles de masa muscular y ósea significativamente mayor que las niñas $(\mathrm{p}<0.05)$. El análisis comparativo respecto a la práctica de actividad física mediante el cuestionario PAQ-A reveló una mayor práctica de actividad física, tanto escolar como extraescolar en los chicos en comparación con las chicas $(p<0.05)$. No se observaron cambios estadísticamente significativos para ninguna de las otras variables estudiadas.

En la Tabla 2 se observan los resultados correspondientes a la distribución de los participantes por sexo atendiendo a la categorización del IMC y del porcentaje graso, en función los valores de referencia. El análisis de distribución de chi-cuadrado no reveló diferencias estadísticamente significativas en cuanto a la categorización del IMC respecto al sexo, aunque existió una tendencia a la significación estadística. La muestra total reveló una prevalencia de sobrepeso del 24-25\% y del 5-15\% de obesidad en ambos sexos. Respecto al porcentaje graso, se observó una prevalencia de sobrepeso del 13-23\% y de obesidad del 13-29\%. El análisis de chi-cuadrado reveló una mayor prevalencia de obesidad en los niños respecto a las niñas $(\mathrm{p}<0.05)$ según el porcentaje graso. En concreto, el ajuste de Bonferroni reveló un mayor porcentaje de niños con obesidad, mientras que las niñas tendieron a presentar un mayor porcentaje de normalidad.

La Tabla 3 muestra la frecuencia de consumo de alimentos en la población de estudio en función del sexo y las recomendaciones establecidas. El análisis de distribución no mostró diferencias estadísticamente significativas en 
cuanto al consumo de alimentos en función del sexo. Una gran cantidad de alumnos, tanto niños como niñas, mostraron una baja frecuencia de consumo de agua (39-48 \% de niños), así como de verduras y frutas (78-82\% de participantes), pescado (48-52\% de participantes) y legumbres (39$46 \%$ de participantes). Por otro lado, encontramos que consumían alimentos ultra procesados no recomendables en cantidades bastante considerables, posibilitando el desplazamiento de otros alimentos muchos más nutritivos y necesarios para cubrir los requerimientos energéticos de los adolescentes. Igualmente, el análisis de distribución de chi-cuadrado no mostró diferencias estadísticamente significativas atendiendo al IMC y el porcentaje de grasa categorizado (datos no mostrados).

Finalmente, la Tabla 4 muestra el análisis comparativo del sexo y el rendimiento académico sobre a la composición corporal, la práctica de AF y la FFQ de alimentos. En referencia al porcentaje graso, los resultados mostraron diferencias estadísticamente significativas siendo mayor en aquellos alumnos con calificaciones de tendencia central (aprobados y notables) que en alumnos con calificaciones más extremas (suspensos y sobresalientes) $(\mathrm{p}<0.05)$. No se encontraron diferencias estadísticamente significativas entre el rendimiento académico y el IMC. En la práctica física escolar y extraescolar, el sexo masculino mostró una mayor práctica de actividad física que las chicas $(\mathrm{p}<0.05)$. Sin embargo, no se encontraron diferencias estadísticamente significativas en-

\begin{tabular}{|c|c|c|c|c|c|c|c|c|}
\hline \multirow{2}{*}{\multicolumn{2}{|c|}{$\begin{array}{l}\text { Tabla } 4 \\
\text { Datos antropométricos, Actividad física } \\
\text { rendimiento académico y el género. } \\
\end{array}$}} & \multirow{2}{*}{\multicolumn{4}{|c|}{ Muestra Total Femenino }} & \multicolumn{2}{|c|}{ Masculino } & \multirow{2}{*}{ P Valor } \\
\hline & & & & & & Media & DT & \\
\hline \multicolumn{9}{|c|}{ Composición Corporal } \\
\hline & Suspenso & 17,46 & 3,92 & 14,37 & 1,72 & 19,00 & 3,85 & 0,042 \\
\hline & Aprobado & 19,07 & 3,64 & 19,36 & 3,66 & 18,79 & 3,71 & 0,653 \\
\hline \multirow[t]{5}{*}{ IMC (Kg/m2) } & Notable & 19,48 & 3,11 & 18,69 & 3,17 & 20,69 & 2,65 & 0,020 \\
\hline & Sobresaliente & 17,48 & 2,39 & 17,43 & 2,77 & 17,53 & 2,02 & 0,940 \\
\hline & $P$ Valor & \multicolumn{2}{|c|}{0,093} & \multicolumn{2}{|c|}{0,076} & & \\
\hline & Suspenso & 20,04 & 5,40 & 19,10 & 3,40 & 20,52 & 6,42 & 0,736 \\
\hline & Aprobado & 24,18 & 7,31 & 26,54 & 6,67 & 21,96 & 7,36 & 0,063 \\
\hline \multirow[t]{3}{*}{ Grasa (\%) } & Notable & 25,68 & 5,15 & 25,43 & 5,20 & 26,07 & 5,17 & 0,661 \\
\hline & Sobresaliente & 22,48 & 5,96 & 24,46 & 5,95 & 19,94 & 5,30 & 0,137 \\
\hline & $P$ Valor & \multicolumn{2}{|c|}{0,038} & \multicolumn{2}{|c|}{0,219} & \multicolumn{2}{|c|}{0,050} & \\
\hline \multicolumn{9}{|l|}{ Actividad Física } \\
\hline & Suspenso & 4,11 & 1,45 & 3,00 & 2,00 & 4,67 & 0,82 & 0,106 \\
\hline \multirow{5}{*}{ AF Escolar } & Aprobado & 4,14 & 1,00 & 3,76 & 1,03 & 4,50 & 0,86 & 0,028 \\
\hline & Notable & 4,21 & 0,91 & 4,06 & 1,01 & 4,43 & 0,68 & 0,152 \\
\hline & Sobresaliente & 4,44 & 0,73 & 4,33 & 0,71 & 4,57 & 0,79 & 0,535 \\
\hline & $P$ Valor & \multicolumn{2}{|c|}{0,765} & \multicolumn{2}{|c|}{0,209} & & & \\
\hline & Suspenso & 3,04 & 1,02 & 2,44 & 1,26 & 3,33 & 0,84 & 0,241 \\
\hline & Aprobado & 3,10 & 0,85 & 2,75 & 0,80 & 3,44 & 0,78 & 0,013 \\
\hline AF Extraescolar & Notable & 3,04 & 0,99 & 2,91 & 0,98 & 3,25 & 1,00 & 0,216 \\
\hline & Sobresaliente & 3,02 & 1,09 & 2,81 & 0,96 & 3,29 & 1,25 & 0,408 \\
\hline & P Valor & 0,9 & 89 & 0,8 & & & & \\
\hline & Suspenso & 3,08 & 0,92 & 2,37 & 1,06 & 3,43 & 0,68 & 0,107 \\
\hline & Aprobado & 2,98 & 0,67 & 2,72 & 0,57 & 3,22 & 0,68 & 0,024 \\
\hline PAQ Total & Notable & 3,04 & 0,73 & 2,90 & 0,74 & 3,23 & 0,68 & 0,109 \\
\hline & Sobresaliente & 3,07 & 0,70 & 2,96 & 0,77 & 3,21 & 0,64 & 0,504 \\
\hline & P Valor & 0,9 & & 0,5 & & & & \\
\hline Frecuencia de Consun & o de Alimentos & & & & & & & \\
\hline & Suspenso & 2,89 & 1,27 & 3,33 & 1,53 & 2,67 & 1,21 & 0,495 \\
\hline & Aprob & 3,57 & 1,09 & 3,59 & 1,06 & 3,56 & 1,15 & 0,931 \\
\hline Agua (r/día) & Notable & 3,68 & 1,11 & 3,84 & 1,05 & 3,43 & 1,16 & 0,184 \\
\hline & Sobresaliente & 4,00 & 1,15 & 4,22 & 0,97 & 3,71 & 1,38 & 0,401 \\
\hline & P Valor & 0,1 & & 0,4 & & & & \\
\hline & Suspenso & 2,67 & 1,50 & 3,00 & 1,73 & 2,50 & 1,52 & 0,668 \\
\hline & Aprobado & 3,80 & 2,00 & 3,82 & 2,10 & 3,78 & 1,96 & 0,947 \\
\hline Verduras/Fruta (r/día) & Notable & 2,94 & 1,54 & 3,00 & 1,65 & 2,86 & 1,39 & 0,744 \\
\hline & Sobresaliente & 2,44 & 1,55 & 1,89 & 1,17 & 3,14 & 1,77 & 0,110 \\
\hline & P Valor & 0,0 & 28 & 0,0 & & & & \\
\hline & Suspenso & 2,00 & 1,50 & 2,00 & 2,00 & 2,00 & 1,41 & 1,000 \\
\hline & Aprobado & 1,66 & 1,16 & 1,88 & 1,11 & 1,44 & 1,20 & 0,271 \\
\hline Legumbres (r/día) & Notable & 1,74 & 1,06 & 1,75 & 1,02 & 1,71 & 1,15 & 0,906 \\
\hline & Sobresaliente & 1,88 & 1,15 & 1,44 & 0,88 & 2,43 & 1,27 & 0,089 \\
\hline & P Valor & 0,8 & 34 & 0,7 & & & & \\
\hline & Suspenso & 2,78 & 1,30 & 3,00 & 1,00 & 2,67 & 1,51 & 0,793 \\
\hline & Aprobado & 1,91 & 1,01 & 1,82 & 1,01 & 2,00 & 1,03 & 0,613 \\
\hline Pan (r/día) & Notable & 2,08 & 1,21 & 1,94 & 1,05 & 2,29 & 1,42 & 0,309 \\
\hline & Sobresaliente & 2,00 & 1,03 & 2,00 & 1,12 & 2,00 & 1,00 & 1,000 \\
\hline & P Valor & 0,2 & 43 & 0,3 & & & & \\
\hline & Suspenso & 1,67 & 0,87 & 1,33 & 1,53 & 1,83 & 0,41 & 0,451 \\
\hline & Aprobado & 2,31 & 1,05 & 2,29 & 0,99 & 2,33 & 1,14 & 0,914 \\
\hline Lácteos (r/día) & Notable & 2,64 & 1,09 & 2,53 & 1,05 & 2,81 & 1,17 & 0,370 \\
\hline & Sobresalie & 2,75 & 0,86 & 2,56 & 0,88 & 3,00 & 0,82 & 0,320 \\
\hline & P Valor & 0,0 & & 0,2 & & & & \\
\hline
\end{tabular}

tre el rendimiento académico y una mayor práctica de actividad física. Finalmente, atendiendo al análisis comparativo entre el sexo, el rendimiento académico y la FFQ de alimentos, se observó que en la muestra total existía un consumo de frutas o verduras y lácteos significativamente menor en aquellos niños cuyo rendimiento académico era peor $(\mathrm{p}<0.05)$.

\section{Discusión}

Los hallazgos del presente estudio sugieren una prevalencia del 24-25\% (sobrepeso) y del 5-15\% (obesidad) con respecto al IMC y del 13-13\% (sobrepeso) y 13-29\% (obesidad) con respecto al porcentaje graso, siendo este último significativamente superior en chicos que en chicas y en sujetos aprobados y notables que aquellos suspensos y sobresalientes. Para la actividad física reportada, los chicos practican más AF que las chicas, además de presentar una mayor masa muscular y ósea. En general, y a pesar de no encontrar relación directa entre el sexo, el IMC o el porcentaje graso con la FFQ, podemos confirmar un consumo elevado de alimentos ultra procesados, desplazando alimentos sumamente necesarios como las frutas, verduras, legumbres o lácteos. Finalmente, y a pesar de la falta de asociación entre el rendimiento académico estratificado y el resto de las variables, observamos que tanto un bajo consumo de alimentos como las frutas y verduras o los lácteos, así como un mayor porcentaje graso parece estar relacionado con un empeoramiento del rendimiento académico. Respecto a la influencia de los hábitos alimentarios sobre el rendimiento académico, encontramos estudios que establecen una relación (Burrows, Goldman, Olson, Byrne, \& Coventry, 2017), aunque también encontramos evidencia que concluye una relación bastante moderada o inexistente (Burrows, Goldman, Pursey, \& Lim, 2017).

En cuanto a la obesidad, diversos estudios realizados a nivel europeo (World Health Organization, 2017; Hu et al., 2017) muestran cifras similares de sobrepeso y obesidad a las obtenidas en el presente estudio. Centrándonos en España, también encontramos estudios relacionados con la prevalencia de sobrepeso y obesidad con cifras similares al nuestro (Antelo, Magdalena, y Reboredo, 2017). A pesar de haber cierta evidencia que establece mayor obesidad y sobrepeso en chicas que en chicos, es muy escasa y no se relaciona con estudios españoles (Wang et al., 2014). La evidencia relacionada con España coincide con el presente estudio en afirmar que los chicos adolescentes presentan mayores niveles de sobrepeso y obesidad que las chicas (Bazán et al., 2017; Inchley et al., 2017).

Más concretamente, $\mathrm{y}$ atendiendo a cuestiones genéticas, los sujetos masculinos suelen presentar un mayor predominio de masa muscular que los sujetos femeninos. Esto suele suceder en todos los rangos de edades, especialmente en adultos (Bredella, 2017), pero también es observable en los adolescentes (Hormazábal-Peralta, Espinoza, Cáceres, y Lizana, 2018). Precisamente, en el estudio de Marwaha et al. (2017), además de un mayor desarrollo muscular en niños que en niñas, se encontraron relaciones positivas entre una mayor masa muscular y una mayor masa ósea siendo directamente proporcional entre ambas (Zhu et al., 2014; Winther et al., 2018). Además, los chicos suelen practicar más AF que 
las chicas, esto parece ser otro factor determinante a la hora de tener mayor masa muscular y ósea (Santos, Elliott-Sale, y Sale, 2017).

En relación a la práctica de AF reportada, nuestro estudio mostró que los chicos presentaban una mayor frecuencia de práctica de AF que las chicas. En concreto, estos registraron una mayor puntuación global en el PAQ-A, además de tener puntuaciones más elevadas referidas a la práctica de AF escolar y extraescolar. En concordancia con nuestros resultados, un estudio reciente llevado a cabo por Beltrán Carrillo et al. (2017), demostró que esta tendencia es similar para los chicos dentro de la misma etapa de maduración. Respecto al horario escolar, Martínez-Gómez et al. (2009) determinaron los niveles de práctica de AF en el recreo, observando al igual que en nuestro estudio, que los chicos presentaban una mayor tasa de actividad que las chicas.

En relación a las pautas alimentarias, en nuestro país disponemos de la dieta Mediterránea como modelo referente de alimentación sana y saludable. Organismos como la SENC se esfuerza para que la sociedad ajuste su alimentación en base al modelo de Pirámide Alimentaria que en la actualidad conocemos (Sociedad Española de Nutrición Comunitaria, 2017). En este documento se informa sobre la FFQ agrupado por tipos de alimentos y su recomendación de consumo habitual. En nuestro estudio, sin embargo, observamos resultados poco esperanzadores en el consumo de alimentos por parte de los adolescentes, ya que por lo general no cumplían con las recomendaciones establecidas. La gran mayoría de participantes presentaron un consumo bajo o moderado de alimentos sumamente importantes como las legumbres, lácteos y, sobre todo, frutas y verduras. Por el contrario, en los alimentos ultra procesados se observó un elevado consumo, superando con creces el consumo ocasional y moderado que se aconseja, y rompiendo así todas las recomendaciones sobre una correcta alimentación. En un reciente estudio (Inchley J et al., 2017) en el que se analizó el consumo de alimentos por parte de adolescentes pertenecientes a 40 países europeos, España ocupaba el último lugar en el consumo tanto de vegetales como simultáneamente el consumo de frutas y vegetales. Por el contrario, no parece extraño si tenemos en cuenta que los alimentos ultra procesados han aumentado considerablemente en nuestro día a día llegando a triplicar su presencia en las listas de la compra (Latasa, Louzada, Steele, y Monteiro, 2017). El consumo de alimentos ultra procesados no solo resulta perjudicial por desplazar otros alimentos mucho más ricos, en cuanto a nutrientes se refiere, sino que también están relacionados con una mayor prevalencia de obesidad y sobrepeso en España (Mendonça et al., 2016), con un mayor porcentaje graso (Costa, DelPonte, Assunção, y Santos, 2018) y con una mayor circunferencia de cintura (Bigornia et al., 2015). A pesar de ello, en nuestro estudio no encontramos relación directa entre el consumo de este tipo de alimentos y un mayor IMC o porcentaje graso en nuestro colectivo de interés.

En referencia al rendimiento académico, quisimos explorar su asociación con el resto de variables. En cuanto a los resultados obtenidos con respecto al IMC, el porcentaje graso y el rendimiento académico, se obtuvieron relaciones significativas entre el porcentaje graso y el rendimiento académico, aunque la relación no se dio a todos los niveles. Exis- ten estudios, aunque pocos, que reflejan una relación positiva entre una mejor composición corporal y un mejor rendimiento académico (Morita et al., 2016), sin embargo, otros estudios no encuentran esta relación (Santana, Hill, Azevedo, Gunnarsdottir, y Prado, 2017). Por tanto, se genera controversia en cuanto a la relación entre estas variables. De la misma manera, en el estudio de la influencia de los hábitos alimentarios sobre el rendimiento académico existen discrepancias respecto a su relación positiva bastante moderada o inexistente (Burrows et al., 2017). En nuestro estudio, a pesar de no obtener relaciones significativas por limitación del tamaño muestral al estratificar por calificaciones, pudimos observar una relación entre mayor práctica física y mayor rendimiento académico en chicas ( $\mathrm{r}=0.249$; $\mathrm{p}=0.049$ ), coincidiendo así con una amplia evidencia existente (Álvarez-Bueno et al., 2017; Hernández y Portolés, 2016). A pesar de la amplia evidencia, conviene reiterar que en este trabajo los resultados no fueron los esperados, y concuerda con otros estudios en los cuales no se ha encontrado una relación positiva o la relación encontrada ha sido bastante moderada (Oliveira et al., 2017).

El presente estudio, por tanto, pone de manifiesto la necesidad de una intervención mediante una educación en hábitos saludables, con la finalidad de fomentar un aumento de la práctica de actividad física, especialmente en niñas, así como unos mejores hábitos alimentarios que, finalmente, podrían tener una posible repercusión sobre el rendimiento académico. El estudio cuenta con ciertas limitaciones. A pesar de que la muestra es considerable y supone dos tercios de la población escolarizada para este rango de edad pertenecientes a la localidad de Bollullos, ha sido elegida de forma no probabilística mediante muestreo intencional. Por otro lado, al tratarse el estudio de carácter transversal, los datos recogidos son de un momento puntual, por lo que las relaciones establecidas pueden no ser del todo rigurosas. Sería conveniente realizar futuras investigaciones donde se controlaran en mayor medida los aspectos comentados anteriormente y observar si los resultados coinciden.

\section{Conclusiones}

En base a lo anteriormente mencionado podemos concluir que los chicos presentan una mayor masa muscular y ósea que las chicas adolescentes, al igual una mayor tendencia a la práctica física. La prevalencia de sobrepeso y obesidad requiere especial consideración, siendo del 24-25\% (sobrepeso) y del 5-15\% (obesidad) con respecto al IMC, y del 13-23\% (sobrepeso) y 13-29\% (obesidad) con respecto al porcentaje graso. Se ha hallado que los escolares de la presente investigación no alcanzan las recomendaciones alimentarias mínimas, con cifras muy bajas de alimentos necesarios como frutas, verduras, lácteos, pescados o legumbres, y un consumo muy excesivo de alimentos insanos y ultra procesados. Un bajo consumo de alimentos como frutas, verduras y lácteos se asoció con un peor rendimiento académico en los escolares.

\section{Agradecimientos}

Los autores agradecen a los colegios participantes del 
estudio: colegios públicos Reyes Católicos y Las Viñas, pertenecientes a Bollullos par del Condado (Huelva).

\section{Referencias}

Abalde-Amoedo, N., y Pino-Juste, M. (2016). Influencia de la actividad física y el sobrepeso en el rendimiento académico: revisión teórica. Sportis, 2(1), 147-161. Doi: https:/ /doi.org/10.17979/sportis.2016.2.1.1446

Álvarez-Bueno, C., Pesce, C., Cavero-Redondo, I., SánchezLópez, M., Garrido-Miguel, M., y Martínez-Vizcaíno, V. (2017). Academic Achievement and Physical Activity: A Meta-analysis. Pediatrics, e20171498. Doi: 10.1542/ peds.2017-1498

Angosto, M. C. (2015). Obesidad: Pandemia del siglo XXI. Monografías de la Real Academia Nacional de Farmacia.

Antelo, M., Magdalena, P., y Reboredo, J. C. (2017). Obesity: A major problem for Spanish minors. Economics and human biology, 24, 61-73. Doi: 10.1016/j.ehb.2016.11.002

Bazán, M. J. A., Trujillo, M. I. J., Wärnberg, J., Fernández, S. D., deAndrés, A. L., y Farinós, N. P. (2017). Differences in the prevalence of diagnosis of overweight-obesity in Spanish children according to the diagnostic criteria set used. Gaceta sanitaria. Doi: 10.1016/j.gaceta.2017.07.014

Beltrán Carrillo, V. J., Sierra, A. C., Jiménez Loais, A., GonzálezCutre, D., Martínez Galindo, C.,y Cervelló, E. (2017). Diferencias según género en el tiempo empleado por adolescentes en actividad sedentaria y actividad física en diferentes segmentos horarios del día. Retos. Nuevas tendencias en Educación Física, Deporte y Recreación, (31).

Benítez-Porres, J., Alvero-Cruz, J.R., Sardinha, L.B., LópezFernández, I., y Carnero, E.A. (2016). Cut-off values for classifying active children and adolescents using the Physical Activity Questionnaire: PAQ-C and PAQ-A. Nutrición Hospitalaria, 33 (5), 1036-1044. Doi: http:// dx.doi.org/10.20960/nh.564.

Bigornia, S. J., LaValley, M. P., Noel, S. E., Moore, L. L., Ness, A. R., y Newby, P. K. (2015). Sugar-sweetened beverage consumption and central and total adiposity in older children: a prospective study accounting for dietary reporting errors. Public health nutrition, 18(7), 11551163. Doi: $10.1017 /$ S1368980014001700

Bredella, M. A. (2017). Sex Differences in Body Composition. In Sex and Gender Factors Affecting Metabolic Homeostasis, Diabetes and Obesity (pp. 9-27). Springer, Cham. Doi: 10.1007/978-3-319-70178-3_2

Burrows, T., Goldman, S., Olson, R. K., Byrne, B., y Coventry, W. L. (2017). Associations between selected dietary behaviours and academic achievement: A study of Australian school aged children. Appetite, 116, 372-380. Doi: 10.1016/j.appet.2017.05.008

Burrows, T., Goldman, S., Pursey, K., y Lim, R. (2017). Is there an association between dietary intake and academic achievement: a systematic review. Journal of Human Nutrition and Dietetics, 30(2), 117-140. Doi: 10.1111/ jhn.12407

Centers for Disease Control and Prevention, 2019. Centers for Disease Control and Prevention, Saving Lives,
Protecting People.Atlanta,USA. Recuperado de: https:// www.cdc.gov/

Costa, C. S., Del-Ponte, B., Assunção, M. C. F., y Santos, I. S. (2018). Consumption of ultra-processed foods and body fat during childhood and adolescence: a systematic review. Public health nutrition, 21(1), 148-159. Doi: 10.1017/S1368980017001331

Gargallo Fernández, M., Quiles Izquierdo, J., Basulto Marset, J., Breton Lesmes, I., Formiguera Sala, X., y Salas-Salvadó, J. (2012). Recomendaciones nutricionales basadas en la evidencia para la prevención y el tratamiento del sobrepeso y la obesidad en adultos (Consenso FESNADSEEDO): La dieta en la prevención de la obesidad (II/ III). Nutrición Hospitalaria, 27(3), 800-832. Doi: 10.3305/ nh.2012.27.3.5678

Guillamón,A. R., Canto, E. G., y López, P. J. C. (2019). Relación entre capacidad aeróbica y el nivel de atención en escolares de primaria (Relationship between aerobic capacity and level of attention in primary school children). Retos, (35), 36-41.

Hernández, J. G., y Portolés, A. (2016). Recomendaciones de actividad física y su relación con el rendimiento académico en adolescentes de la Región de Murcia. Retos: nuevas tendencias en educación física, deporte y recreación, (29), 100-104.

Hormazábal-Peralta, A., Espinoza, J., Cáceres, P., y Lizana, P. A. (2018). Adolescents with high intellectual ability: differences in body composition and physical activity by sex. Nutricion hospitalaria, 35(1), 38-43. Doi: http:// dx.doi.org/10.20960/nh.1170

Hu, L., Huang, X., You, C., Li, J., Hong, K., Li, P., ... y Bao, H. (2017). Prevalence of overweight, obesity, abdominal obesity and obesity-related risk factors in southern China. PloS one, 12(9), e0183934. Doi: 10.1371/ journal.pone.0183934

Iaccarino Idelson, P., Scalfi, L., Valerio, G. (2017) Adherence to the Mediterranean Diet in children and adolescents: A systematic review, Nutrition, Metabolism and Cardiovascular Diseases, 27 (4), 283-299. Doi: 10.1016/ j.numecd.2017.01.002

Inchley, J., Currie, D., Jewell, J., Breda, J., y Barnekow, V. Adolescent obesity and related behaviours: trends and inequalities in the WHO European Region, 2002-2014. Copenhagen, WHO Regional Office for Europe, 2017.

Knox, E., y Muros, J. J. (2017). Association of lifestyle behaviours with self-esteem through health-related quality of life in Spanish adolescents. European journal of pediatrics, 176(5), 621-628. Doi: 10.1007/s00431-0172886-z

Latasa, P., Louzada, M. L. D. C., Steele, E. M., y Monteiro, C. A. (2017). Added sugars and ultra-processed foods in Spanish households (1990-2010). European journal of clinical nutrition, 72(10), 1404-1412. Doi: 10.1038/s41430017-0039-0

Laurson, K. R., Eisenmann, J. C., y Welk, G. J. (2011). Body fat percentile curves for US children and adolescents. American journal of preventive medicine, 41(4), S87-S92. Doi: 10.1016/j.amepre.2011.06.044

Lera, L., Fretes, G., González, C. G., Salinas, J., y Vio, F. (2015). Validación de un instrumento para evaluar consumo, 
hábitos y prácticas alimentarias en escolares de 8 a 11 años. Nutrición Hospitalaria, 31(5), 1977-1988. Doi:10.3305/nh.2015.31.5.8607

Martínez-Gómez, D., Martínez-de-Haro, V., Pozo, T., Welk, G. J., Villagra, A., Calle, M. E., y Veiga, O. L. (2009). Fiabilidad y validez del cuestionario de actividad física PAQ-A en adolescentes españoles. Revista española de salud pública, 83,427-439. Doi: 10.1590/S1135-57272009000300008

Martinez-Gomez, D., Veiga, O. L., Zapatera, B., GomezMartinez, S., Martínez, D., y Marcos, A. (2014). Physical activity during high school recess in Spanish adolescents: the AFINOS study. Journal of Physical Activity and Health, 11(6), 1194-1201. Doi: 10.1123/jpah.2012-0345

Marwaha, R. K., Garg, M. K., Bhadra, K., Mahalle, N., Mithal, A., y Tandon, N. (2017). Lean body mass and bone health in urban adolescents from northern India. Indian pediatrics, 54(3), 193-198.

Mendonça, R. D. D., Pimenta, A. M., Gea, A., de la FuenteArrillaga, C., Martinez-Gonzalez, M.A., Lopes, A. C. S., y Bes-Rastrollo, M. (2016). Ultraprocessed food consumption and risk of overweight and obesity: the University of Navarra Follow-Up (SUN) cohort study, 2. The American journal of clinical nutrition, 104(5), 1433-1440. Doi: 10.3945/ajcn.116.135004

Moreno, L. (2015). La alimentación del adolescente. Nutrición y Salud Mediterráneo Económico, 27, 75-86.

Morita, N., Nakajima, T., Okita, K., Ishihara, T., Sagawa, M., y Yamatsu, K. (2016). Relationships among fitness, obesity, screen time and academic achievement in Japanese adolescents. Physiology y behavior, 163, 161-166. Doi: 10.1016/j.physbeh.2016.04.055

Nagy, P., Kovacs, E., Moreno, L. A., Veidebaum, T., Tornaritis, M., Kourides, Y., Siani, A., Lauria, F., Sioen, I., Claessens, M., Mårild, S., Lissner, L., Bammann, K., Intemann, T., Buck, C., Pigeot, I., Ahrens, W., y Molnár, D. IDEFICS consortium. (2014). Percentile reference values for anthropometric body composition indices in European children from the IDEFICS study. International journal ofobesity, 38(S2), S15. Doi: 10.1038/ijo.2016.119

Organisation for Economic Co-operation and Development (OECD) (2012). Equity and Quality in Education: Supporting Disadvantaged Students and Schools. Paris: OECD. Doi: 10.1007/ springerreference_75881

Oliveira, T., Pizarro, A., Costa, M., Fernandes, L., Silva, G., Mota, J., y Ribeiro, J. C. (2017). Cardiorespiratory fitness, but not physical activity, is associated with academic achievement in children and adolescents. Annals of human biology, 44(4), 309-315. Doi: 10.1080/ 03014460.2017.1308010

Organización Mundial de la Salud. (2018). Actividad física. Datos y cifras. Recuperado de: http://www.who.int/es/ news-room/fact-sheets/detail/physical-activity

Organización Mundial de la Salud, 2017. Salud de la madre, el recién nacido, del niño y del adolescente. Desarrollo en la adolescencia. Recuperado de: http:/www.who.int/ maternal_child_adolescent/topics/adolescence/dev/es/

Pérez-Farinós, N., Villar-Villalba, C., López Sobaler, A. M., Dal Re Saavedra, M. A., Aparicio, A., Santos Sanz, S., Robledo de Dios, T., Castrodeza-Sanz, J. J., y Ortega Anta, R.M.. (2017). The relationship between hours of sleep, screen time and frequency of food and drink consumption in Spain in the 2011 and 2013 ALADINO: a cross-sectional study. BMC public health, 17(1), 33. Doi: 10.1186/s12889016-3962-4

Santana, C. C. A., Hill, J. O., Azevedo, L. B., Gunnarsdottir, T., y Prado, W. L. (2017). The association between obesity and academic performance in youth: a systematic review. Obesity Reviews, 18(10), 1191-1199. Doi: 10.1111/ obr.12582

Santos, L., Elliott-Sale, K. J., y Sale, C. (2017). Exercise and bone health across the lifespan. Biogerontology, 18(6), 931-946. Doi: 10.1007/s10522-017-9732-6

Sociedad Española de Nutrición Comunitaria (2017). Nutricióncomunitaria: Piramide Alimentacion Saludable SENC-Edad escolar. Recuperado de: http:// www.nutricioncomunitaria.org/es/noticia/se-presentanlas-nuevas-guias-alimentarias-para-la-poblacionespanola-elaboradas-por-la-senc-con-la-

Son, W. M., Sung, K. D., Bharath, L. P., Choi, K. J., y Park, S. Y. (2017). Combined exercise training reduces blood pressure, arterial stiffness, and insulin resistance in obese prehypertensive adolescent girls. Clinical and Experimental Hypertension, 39(6), 546-552. Doi: 10.1080/ 10641963.2017.1288742

Tu, H., Wen, C. P., Tsai, S. P., Chow, W. H., Wen, C., Ye, Y., Zhao, H., Tsai, M. K., Huang, M., Dinney, C. P., Tsao, C. K., y Tsao, C. K. (2018). Cancer risk associated with chronic diseases and disease markers: prospective cohort study. BMJ, 360, k134. Doi: 10.1136/bmj.k134

Wang, D., Qian, Z., Wang, J., Yang, M., Lee, Y. L., Liu, F., Liu, M. M., Zhao, Y., Liu, Y. Q., Huang, M. M., Liu, Y., Sun, J., Liu, Y. Z., Wu, C. C., Dong, G. H., y Liu, Y. (2014). Genderspecific differences in associations of overweight and obesity with asthma and asthma-related symptoms in 30056 children: result from 25 districts of Northeastern China. Journal of Asthma, 51(5), 508-514. Doi: 10.3109/ 02770903.2014.892963

Winther, A., Jørgensen, L., Ahmed, L. A., Christoffersen, T., Furberg, A. S., Grimnes, G., Jorde, R., Nilsen, O. A., Dennison, E., y Emaus, N. (2018). Bone mineral density at the hip and its relation to fat mass and lean mass in adolescents: the Tromsø Study, Fit Futures. BMC Musculoskeletal Disorders, 19(1), 21. Doi: 10.1186/ s12891-018-1933-x

World Health Organization. (2017). Tenfold increase in childhood and adolescent obesity in four decades: new study by Imperial College London and WHO. Saudi Medical Journal, 38(11), 1162-1163.

Zhu, K., Briffa, K., Smith, A., Mountain, J., Briggs, A. M., Lye, S., Pennell, C., Straker, L., y Walsh, J. P. (2014). Gender differences in the relationships between lean body mass, fat mass and peak bone mass in young adults. Osteoporosis International, 25(5), 1563-1570. Doi: 10.1007/s00198-014-2665-X

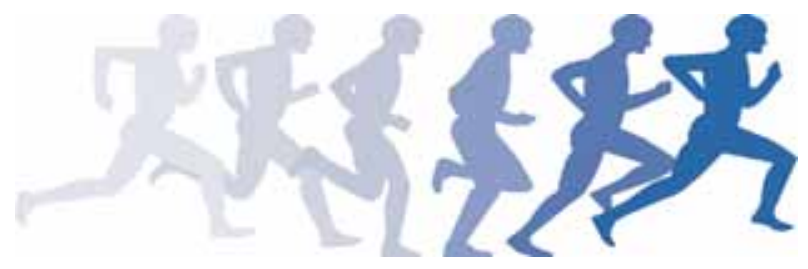

